\title{
Chaotic features in classical scattering processes between ions and atoms
}

\author{
Fabio Sattin ${ }^{\dagger}$ and Luca Salasnich ${ }^{\ddagger}$ \\ † Dipartimento di Ingegneria Elettrica, Università di Padova, \\ via Gradenigo 6/a, 35131 Padova, Italy \\ ‡ Dipartimento di Matematica Pura ed Applicata, Università di Padova \\ via Belzoni 7, 35131 Padova, Italy \\ and \\ Istituto Nazionale di Fisica Nucleare, Sezione di Padova, \\ via Marzolo 8, 35131 Padova, Italy
}

\begin{abstract}
A numerical study has been done of collisions between protons and hydrogen atoms, treated as classical particles, at low impact velocities. The presence of chaos has been looked for by investigating the processes with standard techniques of the chaotic-scattering theory. The evidence of a sharp transition from nearly regular scattering to fully developed chaos has been found at the lower velocities.

PACS numbers: $05.45 .+\mathrm{b}, 34.70+\mathrm{e}$
\end{abstract}

\footnotetext{
${ }^{1}$ E-mail: sattin@pdigi3.igi.pd.cnr.it

${ }^{2}$ E-mail: salasnich@math.unipd.it
} 
Since a long time the use of classical models to study collision processes between atomic particles has been one of the main tools of atomic physics, and among these methods a major role has been played by the Classical Trajectory Monte Carlo (CTMC) method. In this method, originally conceived to study collisions involving an hydrogen atom and a fully stripped ion [1], all the particles involved are treated classically. Randomness is introduced at the level of initial conditions of the motion: electron coordinates are picked up from statistical distributions aiming to mimic quantum ones. At the end of the collision process the electron may be still bound to the original nucleus, possibly in a different quantum state (excitation of the target), it may be bound to the projectile (charge transfer), or may simply be ionized. The physical quantities of interest characterizing the collision (i.e. the cross sections for each process) are obtained by averaging the outcomes over the initial statistical distribution. Along the years the CTMC has been extended and refined to yield an increasing amount of informations (e.g. differential cross sections) as well as to include more complex systems such as multi-electron atoms. For surveys about the subject one can see [2] and references therein. A main limitation of CTMC is that its validity is theoretically justified only for collision energies not too low (the natural unit of measure for velocities in this system is given by the electron classical orbital velocity $v_{e}$, which is the Bohr velocity, or about $2.210^{6} \mathrm{~m} / \mathrm{s}$, for an $\mathrm{H}$ atom in its ground state). In defect of this criterion quantum effects are thought to deeply influence the dynamics through tunnelling of the electron between the nuclei. Since the method shows great simplicity and easiness of use if compared to fully quantal calculations it is tempting to extend it also in this "forbidden" region. 
Recently Keller et al [3] have shown that quantum effects in the dynamical evolution of the electron can satisfactorily be simulated by a stochastic algorithm which adds small random gaussian fluctuations to its otherwise deterministic trajectory. It is shown that: i) the new algorithm improves the results of the standard CTMC as compared to experiment and to fully quantum mechanical calculations at low energies, and ii) the final results are quite insensitive to the details of the perturbation.

However, in spite of the practical effectiveness of the method, yet no sound theoretical reason exists to account for this robustness. Recently, Katsonis and Varvoglis [4] have suggested that the key may be found in the structure of the phase space of the system: if all or a great part of it is chaotic then it is known that such a system is stable against perturbations, namely small modifications of the Hamiltonian do not change the qualitative behaviour of its dynamics. As long as quantum mechanical features can be seen as "perturbations" to the classical Hamiltonian, the same argument justifies the effectiveness of the classical method in front of the experimental results. The validity of this argument relies on the phase space to be entirely or mostly chaotic. If large regions of ordered motion exist then perturbations can destroy them and this will be reflected into final averages. In [4] it is suggested that this hypothesis may be verified by numerically investigating the phase space in search of chaotic regions. The scope of this paper is exactly to start an analysis into this direction.

Since the dimension of the phase space is rather large a complete study is difficult to perform and we have not attempted it: instead of a systematic investigation we have carried out a set of calculations searching for presence of 
traces of chaos. For this reason we have also partially sacrificed the physical soundness of the model by inspecting the region of very low impact energies $(v \ll 1)$ where chaos is more likely to be found but where the classical approximation loses most of its validity. It has to be remarked that even to get the results displayed below it took several hundred hours of CPU time on a DEC ALPHA 2100 workstation.

In the following we will use atomic units, where the velocities are measured in units of the Bohr velocity $v_{e}$.

The system is initially prepared with the target nucleus, a proton, in the origin of coordinates with null velocity, and with the electron orbiting around it. Initial electron coordinates (position and momentum) satisfy the condition that its total energy must be -0.5 units (the binding energy of a $1 \mathrm{~s}$ electron). The proton projectile must start from a large distance ( $>10$ units in our runs) in order that its initial interaction with the target be considered negligible. The equations of the motion are numerically integrated using a fourth order Runge-Kutta-Merson method [5] until the two nuclei are well far apart. The final state of the electron-ionized, captured from the projectile, or simply excited - is determined from standard procedures $[1,6]$. The problem is a standard one of chaotic scattering: we have a dynamical system free to evolve from a given initial state (which is characterized by an input variable, or set of variables, $\theta_{i}$ ), and look for the value of the final state $\theta_{f}$ as a function of $\theta_{i}$. Usually $\theta_{f}$ is a smooth function of $\theta_{i}$ apart possibly a number of singular points: in any arbitrarily small neighborhood of the singularities the output variable varies wildly. The chaotic regions of the phase space are responsible for the singularities: while always of Lebesgue 
measure zero, the set of singularities has a fractal dimension greater than zero in correspondence of a chaotic region (for a complete discussion about the argument, see the reviews of Eckhardt [7] and of Ott and Tél [8]).

The determination of the fractal dimension is based upon the uncertainty exponent technique developed by Bleher et al $[9,10]$. In this work we have kept fixed the initial electron coordinates $(\vec{r}, \vec{p})$, the initial internuclear distance and the relative speed, and the only free parameter left is the impact parameter $b$. By this procedure the dimension of the phase space is reduced to one. The system is let to evolve and the outcome is recorded. Then the procedure is repeated using a slightly different impact parameter, $b \pm \epsilon$, and the results of the two runs are compared. The final state is said uncertain -after Bleher et al [9]-if the two results are different. If we label each of the possible outcomes with an integer number, and plot them $v s b$, in correspondence of an uncertain trajectory we have a discontinuity of the output variable. Some plots of this kind are shown in Figure 1.

We have computed the fraction $f(\epsilon)$ of uncertain trajectories as a function of the parameter $\epsilon$ : it is known that

$$
f(\epsilon) \propto \epsilon^{\alpha} \quad \text { for } \epsilon \rightarrow 0,
$$

and $\alpha$ is related to the boundary fractal dimension (capacity dimension) $d$ through $\alpha=D-d$, where $D$ is the dimension of the parameter space (here, $D=1$ ) $[9,10]$. If the scattering is regular $d=0$ and $\alpha=1$, while in presence of fully developed chaos $d=1$ and $\alpha=0$.

We have first made a scan over impact parameter for different velocities in order to identify the chaotic regions. In Figure 1 the results are plotted for 
one value of $v$ and zooming on $b$. We have arbitrarily assigned the value 1 if at the end of the collision the electron remains bound to the target nucleus, 2 if it is ionized, and 3 if it is bound to the projectile nucleus.It is quite clear that a kind of self-similarity appears. Similar plots at nearly equal velocities show all the same features which disappear at higher values of $v$.

Figure 2 shows $f(\epsilon)$ vs $\epsilon$ for some different velocities. At the lower velocities two different regions are quite clearly visible: one, for $\epsilon \rightarrow 0$, where there is a clear power-law behaviour, and another, at greater $\epsilon$. The correction at great $\epsilon$ is due to a logarithmic term [11],

$$
f(\epsilon) \propto \epsilon^{\alpha}\left(\ln \left(\frac{1}{\epsilon}\right)\right)^{\beta}
$$

which does not affect the determination of the capacity dimension. In [11] a similar behaviour has been associated with a case of nonhyperbolic chaotic scattering, in which the fractal dimension is $d=1$.

In the calculation of the capacity dimension we have fitted the data with simple power-law curves, and skipped the range of $\epsilon$ where the power-law behaviour is not dominant.

In Figure 3, which represents the main result of this work, $d$ is plotted vs impact velocity. One observes a sharp transition between a situation of nearly absence of chaos and one of fully developed chaos at a velocity $v_{c}$ of about 0.06 units. Within statistical errors we cannot state whether there is or not chaos beyond $v_{c}$ : further studies are presently being carried on. This situation resembles that illustrated in [9,11-13] of abrupt transitions from a scattering regime to another. From Figure 2 we see that the transition to non chaotic scattering is associated with an increasing of the crossover 
region, where the logarithmic term in equation (2) is not negligible. On the contrary, at higher velocities, above the transition, no logarithmic behaviour is visible. The critical value of the transition corresponds to an impact energy of about $E=100 \mathrm{eV}$, but we are still not able to associate a clear meaning to this energy. The value of $v_{c}$ is much below that studied in [3] $\left(v_{c}>0.6\right)$ so the present results cannot support the hypoteses of [4]. However presence of chaos at higher velocities cannot absolutely be excluded on the basis of our investigations.

The phase space associated to electronic coordinates obviously plays a crucial role in determining the dynamics of the scattering. We have done several simulations in this sense, varying the initial coordinates $(\vec{r}, \vec{p})$; our computations have not allowed us to see any difference: the functional dependence is the same even if absolute values of $f$ are different.

From our numerical calculations we can conclude that there is chaos in classical processes of charge exchange: it appears in the form of a sudden transition from fully developed chaos to regular motion when increasing the velocity.

We remark that even if the original motivation for this work was about the connections between chaos and the CTMC method, our final results cannot have a direct relevance to CTMC due to the consideration of low impact velocities.

Finally, we point out that up to now only the classical theory has been dealt with but some of the results previously shown may be useful in the quantum theory of chaos: actually the $H$-proton scattering at these low energies is perfectly amenable to quantum calculations so we have an example 
of quantum system whose classical counterpart exhibits chaos, the study of these systems being an active area of research.

F.S. has been financially supported during this work by a grant of the Italian MURST. Computing facilities have been made available by the Istituto Gas Ionizzati del CNR of Padova. 


\section{References}

[1] R. Abrines and I.C. Percival, Proc. Phys. Soc. 88, 861 (1966); R. Abrines and I.C. Percival, ibid. p. 873

[2] P.T. Greenland, Phys. Rep. 81, 131 (1982); B.H. Bransden and M.R.C.

McDowell, Charge Exchange and the Theory of Ion-Atom Collisions, Oxford Science Publications (1992), chap. 8

[3] S. Keller, H. Ast and R.M. Dreizler, J. Phys. B: At. Mol. Opt. Phys. 26, L737 (1993)

[4] K. Katsonis and H. Varvoglis, J. Phys. B: At. Mol. Opt. Phys. 28, L483 (1995)

[5] Subroutine D02BAF, The NAG fortran Library, Oxford

[6] K. Tökesi and G. Hock, Nucl. Instrum. Meth. Phys. Res., Sect. B 86, 201 (1994)

[7] B. Eckhardt, Physica D 33, 89 (1988)

[8] E. Ott, T. Tél, CHAOS 3, 417 (1993)

[9] S. Bleher, C. Grebogi, E. Ott, and R. Brown, Phys. Rev. A 38, 930 (1988); S. Bleher, E. Ott and C. Grebogi, Phys. Rev. Lett., 63, 919 (1989); S. Bleher, C. Grebogi and E. Ott, Physica D 46, 87 (1990)

[10] C. Grebogi, S.W. McDonald, E. Ott, and J.A. Yorke, Phys. Lett. A 99, 415 (1983); S.W. McDonald, E. Ott, and J.A. Yorke, Physica D 17, 125 (1985)

[11] Y.-T. Lau, J.M. Finn, and E. Ott, Phys. Rev. Lett. 66, 978 (1991)

[12] Y.-C. Lai, C. Grebogi, R. Blümel, and I. Kan, Phys. Rev. Lett. 71, $2212(1993)$ 
[13] M. Ding, C. Grebogi, E. Ott, J.A. Yorke, Phys. Rev. A 42, 7025 (1990) 


\section{Figure Captions}

Figure 1: scattering outcome vs impact parameter $b$. Results are labelled according to: excitation, 1; ionization, 2; charge transfer, 3. From the top to the bottom successive blowups are shown. Respectively 1000, 600, and 700 points have been plotted.

Figure 2: plot of $f(\epsilon)$ vs $\epsilon$. Squares: $v=0.0282$; stars: $v=0.0447$; triangles: $v=0.2$.

Figure 3: capacity dimension $d$ vs impact velocity $v$. Error bars are the errors on the slope of the straight lines $\ln f v s \ln \epsilon$ as calculated by linear fits. 